\title{
A Retrospective Analysis of Children with Lower Airway Malacia in a Tertiary Paediatric Hospital
}

\author{
Fazilcan Zirek ${ }^{1}$, Gizem Ozcan ${ }^{1}$, Ozlem Selvi Can ${ }^{1}$, and Nazan Cobanoglu ${ }^{1}$ \\ ${ }^{1}$ Ankara University Faculty of Medicine
}

September 17, 2020

\begin{abstract}
Background: Airway malacia is a condition of excessive airway collapsibility, which causes expiratory reduction in the crosssectional luminal area during respiration. As this disorder may lead to life threatening events and even death in children, it is important to recognise and treat it. Aims: In this study, we aimed to evaluate clinical and radiological features, prognoses, and associated disorders of the patients with lower airway malacia. Methods: A total of 65 patients with lower airway malacia diagnosed by flexible bronchoscopy were included in this study. Demographic and clinical features, radiological findings, video image records of bronchoscopy and prognoses of the patients were evaluated retrospectively. Results: Lower airway malacia was diagnosed in $65(16.6 \%)$ children with a median age of 1 year and 2 months (range: 1 month- 16 years and 8 months). Thirty-five $(53.8 \%)$ of them were male. The numbers of children with isolated tracheomalacia, isolated bronchomalacia and tracheobronchomalacia were nine (13.8\%), 48 (73.8\%) and eight (12.3\%), respectively. The most common reason for admission was recurrent and/or prolonged respiratory infection $(46.2 \%)$ and the most common physical examination finding was stridor (36.9\%). Fifty-eight $(89.2 \%)$ patients had other co-morbidities associated with airway malacia. Inhaled ipratropium bromide therapy was started for $37(56.9 \%)$ patients. Ten $(15.4 \%)$ patients required continuous positive pressure support.. Conclusion: Lower airway malacia is an important disorder in children with respiratory problems and flexible bronchoscopy is a valuable diagnostic method. Sharing experiences in terms of diagnosis and treatment modalities would help patients as well as clinicians.
\end{abstract}

\section{Introduction:}

Airway malacia is a condition of excessive airway collapsibility, which causes expiratory reduction in the cross-sectional luminal area during respiration. Lower airway malacia is classified as tracheomalacia (TM), bronchomalacia (BM) or tracheobronchomalacia (TBM) according to the localization of the malacic segment. Clinical presentation includes stridor or wheeze, brassy cough, recurrent or prolonged respiratory infections, atelectasis and even near-death attacks such as dying spell ${ }^{1}$.

Although this disorder has been known for a long time in paediatric patients, the true incidence of lower airway malacia is still not well known. With increasing bronchoscopic evaluation in paediatric patients, developing devices of suitable size for small children and using advanced imaging methods, lower airway malacia disorders are becoming well recognized. Nevertheless, there is no universally agreed gold standard diagnostic test for lower airway malacia.

After diagnosing a child with malacia, clinicians encounter new challenges, as there is no consensus on disease management related to scarcity of the evidence-based studies in the literature. While tracheostomy with or without invasive mechanical ventilation (MV) was the mainstay of treatment for severe TM in the past, this treatment modality was recently replaced mostly by medical therapy with non-invasive positive pressure support.

In this study, we aimed to review the medical data of the patients with lower airway malacia diagnosed by flexible bronchoscopy and followed in our centre, in terms of clinical and radiological features, prognoses, 
and associated disorders of the patients.

\section{Materials and Methods:}

This retrospective study was approved by the Ethics Committee of our institution.

Patients

In accordance with American Thoracic Society (ATS)/European Respiratory Society (ERS) guideline, 390 patients, who were admitted or consulted to our clinic between June 2014 and June 2020, underwent flexible fiberoptic bronchoscopy (FB) and $410 \mathrm{FB}$ procedures were performed ${ }^{2,3}$. Sixty-five patients were diagnosed with lower airway malacia. Written informed consent was obtained from the families of all patients before bronchoscopy. Demographic and clinical features, radiological findings, video image records of bronchoscopy and prognoses of the patients with lower airway malacia were evaluated retrospectively.

\section{Flexible fiberoptic bronchoscopy}

Flexible fiberoptic bronchoscopy was performed in the operating room with using Olympus CV-190 with CLV -190 EVIS EXERA III (Olympus, New Hyde Park, NY, USA). In patients with suspected laryngomalacia, primarily upper airway was evaluated. Deep sedation was achieved with combination of ketamine and propofol following routine monitoring. Spontaneous breathing was maintained under oxygen support and respiratory support was provided with a face mask, if needed. After evaluating upper airway, general anaesthesia with spontaneous ventilation was achieved with laryngeal mask airway and total intravenous anaesthesia with propofol without neuromuscular agent. While the bronchoscope with an outer diameter of $3.8 \mathrm{~mm}$ was used in patients whose body weights were [?]15 $\mathrm{kg}$, the bronchoscope with an outer diameter of $4.8 \mathrm{~mm}$ was used in heavier patients. During the procedure, $0.5-1 \mathrm{ml} \% 2$ lidocaine with saline solution was given at the level of the vocal cord and the main carina. Video images of all patients were recorded.

\section{Defining and classifying airway malacia}

Airway malacia was defined as greater than $25 \%$ expiratory reduction in the cross-sectional luminal area during quiet respiration on the visual inspection at bronchoscopy ${ }^{4}$. Lower airway malacia was classified as $\mathrm{TM}$, if the collapsibility was located on trachea, or BM, if the collapsibility was restricted to one or both of the mainstem bronchi and/or their divisions at the lobar or segmental level. When at least one of the bronchi affected with trachea, the condition was called TBM ${ }^{1}$. In cases in where TBM didn't exist, the malacia was defined as isolated TM or isolated BM. The localization and severity of TM were assessed with bronchoscopy. Trachea was divided into three regions and classified as upper (from the cricoid to the thoracic inlet), middle (from the thoracic inlet to the mid portion to the intrathoracic trachea) and lower (from the middle of the intrathoracic trachea to the carina) trachea ${ }^{1}$. The severity of TM was described as mild (25$50 \%$ reduction), moderate $(50-90 \%$ reduction) and severe ( $>90 \%$ reduction). The causes of airway malacias were classified according to ERS guideline ${ }^{1}$.

\section{Bronchoalveolar lavage procedure}

Bronchoalveolar lavage (BAL) was performed in patients in cases of suspected respiratory infection to determine the offending microbe, suspected pulmonary hemosiderosis, aspiration pneumonia, pulmonary alveolar proteinosis and unclear diagnosis. All BAL samples were collected and analysed in accordance with the recommended standard procedure of ATS/ERS guideline ${ }^{2,3}$. BAL fluid samples with greater than 25

neutrophils and lower than 10 epithelial cells per microscopic field are considered to be relatively free of contamination. 104 colony forming units per millilitre of BAL fluid are considered to indicate established bacterial infection ${ }^{2}$. BAL fluid results, which did not meet these criterions, were excluded.

\section{Co-morbidities associated with airway malacia}

Co-morbid disorders associated with airway malacia were recorded. In patients, who were indicated, the diagnosis of gastroesophageal reflux disease (GERD) was made by barium oesophagography and/or 24hours oesophageal ph monitoring. For diagnosis of swallow dysfunction, the patients were underwent video 
fluoroscopic swallowing study.

Statistical analysis

Descriptive statistics were expressed as median (interquartile range). Categorical variables were presented as n (\%).

\section{Results:}

\section{Patient characteristics}

In a total of 390 patients and 410 bronchoscopies, lower airway malacia was diagnosed in 65 (16.6\%) children with a median age of 1 year and 2 months (range: 1 month-16 years and 8 months) between June 2014 and June 2020. Thirty-five (53.8\%) of them were male.

\section{Flexible fiberoptic bronchoscopy findings}

The numbers of children with isolated TM, isolated BM and TBM were nine (13.8\%), 48 (73.8\%) and eight $(12.3 \%)$, respectively. The distribution of age and type of airway malacia are shown in Figure $\mathbf{1}$ and the localizations of airway malacias are shown in Table $\mathbf{1}$. Fifteen patients $(23.1 \%)$ had associated laryngomalacia. Localizations with severity of airway collapse in tracheomalacias are shown inTable 2. Among all bronchomalacias, $32(57.1 \%)$ were detected solely on the right lung, $15(26.8 \%)$ solely on the left lung and 9 (16.1\%) on both. One patient had tracheal bronchus and isolated BM was located on this. In 27 (41.5\%) patients, there were co-existing pathological findings: variation of bronchial opening (n:15), granulation tissue (n:10), tracheal and/or bronchial stenosis (n:9), subglottic stenosis (n:4), tracheoesophageal fistula (TOF) opening (n:2), nasopharyngeal collapse (n:1) and complete tracheal ring (n:1).

\section{Clinical features}

The distinction of cause of admission and physical examination findings according to the type of malacia is shown in Table 3 . The most common complaint during admission to our clinic was recurrent and/or prolonged respiratory infection $(46.2 \%)$ and the most common physical examination finding was stridor (36.9\%). A patient with immunodeficiency, who was performed bronchoscopy for microbiological sampling, due to a short-time lower respiratory tract infection, was diagnosed malacia incidentally.

\section{$B A L$ results}

Twelve $(22.6 \%)$ of 53 patients in whom BAL was obtained had a positive bacterial culture. Klebsiella Pneumoniae and Pseudomonas Aeruginosa were the most commonly isolated microorganisms.

\section{Radiographic findings}

Posteroanterior and lateral chest X-ray was performed for all patients before bronchoscopy. While chest radiographs of $19(29.2 \%)$ patients were normal, infiltration was observed in $31(47.7 \%)$ patients and atelectasis was observed in $24(36.9 \%)$ patients on at least one malacic area. In addition to FB and planar graph, 56 $(86.2 \%)$ patients were also examined with thoracic CT. Radiographic findings on thoracic CT are shown in Table 4 . Of ten (15.4\%) patients who had narrowing of the malacic airway, 5 of them had compression on the airways; which included the compression of cardiac and/or vascular structure (n:3), enlargement lymph node ( n:1) and haemangioma (n:1).

\section{Co-morbidities associated with airway malacia}

Fifty-eight (89.2\%) patients had other co-morbidities associated with airway malacias. Type of airway malacia and association with other conditions was shown in Table $\mathbf{5}$. Congenital lung disease includes congenital diaphragmatic hernia, congenital lobar emphysema and mature cystic teratoma. Of $3(4.6 \%)$ oesophageal atresia, 2 were associated with TOF, while one was isolated. Four patients $(6.2 \%)$ had both GERD and swallow dysfunction. Diagnosis of dysmorphic patients were Down Syndrome, Pierre Robin Syndrome, DiGeorge Syndrome, Silver Russell Syndrome, Noonan Syndrome, VACTERL association and Chromosome 14q Terminal Deletion Syndrome. Eight (12.3\%) patients were classified as primary idiopathic 
airway malacia. Twenty-two (33.8\%) patients were born before 37 weeks and eight of them had a history of bronchopulmonary dysplasia (BPD). Forty-six patients with malacia $(70.8 \%)$ had a paediatric and/or neonatal intensive care unit (ICU) hospitalization history. Among those patients with ICU hospitalization history, 31 (67.3\%) patients had invasive mechanical ventilation (MV) and one (2.1\%) had non-invasive MV treatment.

\section{Treatment and follow-up}

The median follow-up period of the patients was 8 months (range: 1 month- 5 years and 9 months). Thirtythree $(50.8 \%)$ patients continued to follow up in our clinic. Inhaled ipratropium bromide therapy was started for $37(56.9 \%)$ patients and terminated after 6 months in four patients because of clinical improvement yet the others continued to treatment. While $20(30.8 \%)$ patients needed supplemental oxygen therapy after diagnosis of malacia, 10 (15.4\%) patients required continuous positive pressure support with invasive MV. Only one patient needed non-invasive MV treatment. Tracheostomy was performed in 10 (15.4\%) patients who were underwent invasive MV treatment; of them, two had isolated TM and one had TBM. None of the patients was performed any surgical treatment for malacic airways. Three patients (4.6\%) died during follow-up.

\section{Discussion:}

With increasing use of bronchoscopic examination in paediatric patient and developing devices of suitable size for small children, airway malacia disorders are becoming well recognized. However, the true incidence of airway malacia is not well known. According to the English literature, two studies that enrolled the highest numbers of patients revealed that the incidence of airway malacia among patients underwent bronchoscopy is $16.6 \%$ and $34 \%$. In the latter, authors included patients with laryngomalacia in their study ${ }^{5,6}$. We found the incidence rate similar to the first study (16.6\%). There are several reasons why the true incidence is not well known. There is no standardized diagnostic criterion of airway malacia. In 2019, ERS Task force report is suggested definition of airway malacia as greater the $50 \%$ expiratory reduction in the cross-sectional luminal area during quiet respiration; but some centres has used the $25 \%$ or $33 \%$ expiratory reduction for diagnosis ${ }^{4}$. Furthermore, in some paediatric pulmonology centres, suitable bronchoscopy device for small infants or new-borns may not be available and lack of device may cause the diagnosis to be delayed or missed. Deficient knowledge or lack of suspicion of general practitioners, even of some paediatricians, about airway malacia may also cause the patients to be undiagnosed. A significant amount of patients with lower airway malacia might be misdiagnosed as asthma.

The type and onset of symptoms depend on localization and severity of the malacic segment. We did not evaluate the onset time in our study, but we found that the most common clinical symptom was recurrent and/or prolonged respiratory infection. The high percentages of respiratory infections in our study may be related to the higher number of cases with BM. Airway malacia is a frequent bronchoscopic finding in children with recurrent respiratory infection and protracted bacterial bronchitis, although it is not known exactly whether malacia is cause or result of respiratory infection ${ }^{7,8}$. Only five of the BM cases with a history of recurrent and/or prolonged respiratory infection had positive BAL culture, and that represents $10.4 \%$ of all BM cases. Overall, $22.6 \%$ of BAL samples were culture positive. The cause of such a low rate may be related to take BAL samples while some of the patients were under antibiotic treatment.

The most common physical examination finding was stridor in our study. Stridor is mainly related to narrowing of extra thoracic airway. Although the most common airway malacia type was BM in our study, the high frequency of stridor might be related to accompanying laryngomalacia.

Airway malacias are classified as primary (congenital) and secondary (acquaried) malacia according to time of abnormal formation on the airways ${ }^{4}$. Although no etiological factor could be found for eight patients to develope malacia in our study, it is difficult to classify them as primary airway malacia. Since, detecting persistence of a malacia during bronchoscopy despite proper treatment is necessary to name a malacia as primary. However, we did not perform any bronchoscopy after treatment to see whether malacia was persisting or not. Therefore, we did not make such a classification in our study. 
In our study, the most common diseases associated with airway malacias were GERD and swallowing dysfunction. The relationship between GERD and airway malacia has been shown in several studies. The high prevalence of gastroesophageal reflux among infants with airways malacia can be related to the negative intrapleural pressure induced by airflow obstruction during respiration and this pressure leads to a malfunction of the lower gastroesophageal sphincter and causes reflux. It is also shown that after antireflux therapy; respiratory symptoms were decreased. The improvement may be related to preventing chronic injury to the lung or it may have occurred spontaneously. ${ }^{9,10}$. More detailed research is required to show this difference. Swallowing dysfunction is also common among airway malacias, or maybe we should say airway malacias are common in patients who have swallowing dysfunction. Although the reason of this association is not well known, it might be related to chronic inflammation of airways due to chronic aspiration.

There is no universally agreed gold standard diagnostic test for airway malacias; but flexible bronchoscopy is the most commonly used diagnostic modality. However, bronchoscopy is an invasive and subjective procedure. Furthermore, it increases end expiratory airway pressure and it can be difficult to detect some mild malacias during bronchoscopy. Chest CT is a valuable test to diagnose respiratory diseases. It could assist to find secondary factors causing malacia after diagnosis or show supporting findings such as stenosis, increased/decreased inflation. In recent years, contrast enhanced multi-detector computed tomography (MDCT) has become an alternative modality to aid in the diagnosis of airway malacia in children by obtaining information about the calibre change of the large airways at both end-inspiration and end-expiration (paired end-inspiratory and end-expiratory) ${ }^{11}$. However, it is very difficult to apply this dynamic technique in paediatric patients, and it is available in limited numbers of tertiary centres. Although we diagnosed airway malacia by performing flexible bronchoscopy, 56 patients were also performed thoracic non-dynamic $\mathrm{CT}$ and 10 of them revealed narrowing of the airway on malacic region.

Previously airway malacia, especially congenital form, has been thought to be self-limited and improved by the second year of life without intervention ${ }^{12}$. Although the majority of the patients were under two years old, none of them were followed without any intervention in our study.

Treatment of malacia depends on the aetiology and severity of the airway collapse. Treatment options include medical and surgical therapy, chest physiotherapy and ventilator support. However, there are no randomised-controlled studies for treatment in airway malacia.

One of the leading medical therapies in malacia is inhaled ipratropium bromide. Although its mechanism is not fully known, it is thought to have an effect on airway muscular tone. In recent animal and human studies showed airway dynamics of trachea are affected by tracheal smooth muscle contraction by cholinergic stimulation $^{13,14}$. Although inhaled ipratropium bromide is an anticholinergic agent, it stimulates smooth muscle contraction by blocking presynaptic muscarinic receptors (M2), which provide feedback inhibition for acetylcholine release in the neuromuscular junction, in low doses ${ }^{15}$. Relying on this effect, inhaled ipratropium bromide therapy was started in patients with malacia in our clinic. However, reliable data could not be obtained from our hospital records about the duration of inhaled ipratropium bromide therapy that improved the symptoms of the patients. In addition, none of the patients underwent control bronchoscopy to assess the improvement in the malacic region. There is only one study in the literature about the effect of 6 months of atropine treatment on clinical improvement; but no data were available when the treatment should be terminated ${ }^{16}$.

Tracheostomy with or without long-term invasive MV was the mainstay of treatment for severe TM in the past. However, because of its life-threatening complications and remarkable advance in non-invasive therapies, tracheostomy without invasive MV is currently used as a last resort. In our study, tracheostomy was performed in $15 \%$ of the patients due to need for invasive MV however, invasive MV indication was not only airway malacia but also respiratory failure related to associated conditions in all of them. Non-invasive MV is another treatment method of malacia. Positive pressure creates a pneumatic stent and prevents the collapse of the airway during respiration ${ }^{4}$. Although, only one patient, who had severe bronchomalacia on bilateral main bronchus, was treated with non-invasive MV in our study. we believe that, it will be the first treatment of choice for severe malacia in the future. 


\section{Limitations of the Study:}

Our study has some limitations. First of all, it is a retrospective study. However, in some instances, when clinical trials are not possible, retrospective studies are available to compare different treatments, define prognostic factors, helpful to assess the feasibility of prospective studies and to help in their design. Secondly, as only two sizes of flexible bronchoscopes (with outer diameters 3.8 and $4.8 \mathrm{~mm}$ ) were available in our centre, we could not evaluate young infants and newborns with bronchoscopic examination and that may affect the results hence incidence of malacia. Thirdly, after starting a treatment type the patients were evaluated only with clinical improvement to figure out the effect of the treatment modality and to decide to terminate it. However, determining the effect of the treatment with bronchoscopic evaluation might be more valuable and objective.

\section{Conclusion:}

Lower airway malacia is an important disorder in children with respiratory problems and flexible bronchoscopy is a valuable diagnostic method. In patients with atypical respiratory findings and no response to treatment, lower airway malacia should be kept in mind. Sharing experiences in terms of diagnosis and treatment modalities would help patients as well as clinicians.

\section{References:}

1. Wallis C, Alexopoulou E, Antón-Pacheco JL, Bhatt JM, Bush A, Chang AB, Charatsi AM, Coleman C, Depiazzi J, Douros K, et al. ERS statement on tracheomalacia and bronchomalacia in children. European Respiratory Journal 2019;54(3).

2. Faro A, Wood RE, Schechter MS, Leong AB, Wittkugel E, Abode K, Chmiel JF, Daines C, Davis S, Eber E, et al. Official American Thoracic Society technical standards: flexible airway endoscopy in children. American journal of respiratory and critical care medicine 2015;191(9):1066-80.

3. Midulla F, De Blic J, Barbato A, Bush A, Eber E, Kotecha S, Haxby E, Moretti C, Pohunek P, Ratjen F et al. Flexible endoscopy of paediatric airways. European Respiratory Journal 2013;22(4):698-708.

4. Rozycli HJ, Houten MLV, Elliott GR. Quantitative assessment of intrathoracic airway collapse in infants and children with tracheobronchomalacia. Pediatr Pulmonol. 1996;21:241-45.

5. Masters IB, Chang AB, Patterson L, Wainwright C, Buntain H, Dean BW, Francis PW. Series of laryngomalacia, tracheomalacia, and bronchomalacia disorders and their associations with other conditions in children. Pediatric pulmonology 2002;34(3):189-95.

6. Pan W, Peng D, Luo J, Liu E, Luo Z, Dai J, Fu Z, Li Q, Huang Y. Clinical features of airway malacia in children: a retrospective analysis of 459 patients. International journal of clinical and experimental medicine 2014;7(9):3005.

7. Chang AB, Gaffney JT, Eastburn MM, Faoagali J, Cox NC, Masters IB. Cough quality in children: a comparison of subjective vs. bronchoscopic findings. Respir Res 2005;6:3.

8. Kompare M, Weinberger M. Protracted bacterial bronchitis in young children: association with airway malacia. J Pediatr 2012;160(1): 88-92.

9. Callahan CW. Primary tracheomalacia and gastroesophageal reflux in infants with cough. Clin Pediatr (Phila) 1998;37:725-32.

10. Bibi H, Khvolis E, Shoseyov D, Ohaly M, Dor DB, London D, Ate D. The prevalence of gastroesophageal reflux in children with tracheomalacia and laryngomalacia. Chest 2004;119(2):409-13.

11. Fraga, J. C., Jennings, R. W., \& Kim, P. C. (2016, June). Pediatric tracheomalacia. In Seminars in pediatric surgery (Vol. 25, No. 3, pp. 156-164). WB Saunders

12. Pan W, Peng D, Luo J, Liu E, Luo Z, Dai J, Fu Z, Li Q, Huang Y. Clinical features of airway malacia in children: a retrospective analysis of 459 patients. International journal of clinical and experimental medicine 2014;7: 3005-12.

13. Koslo RJ, Bhutani VK, Shaffer TM. The role of tracheal smooth muscle contraction on neonatal tracheal mechanics. Pediatric research 1986;20(12):1216-20.

14. Panitch HB, Keklikian EN, Motley RA, Wolfson MR, Schidlow DV. Effect of altering smooth muscle tone on maximal expiratory flows in patients with tracheomalacia. Pediatric pulmonology 1990;9(3):170- 
76.

15. Patel HJ, Barnes PJ, Takahashi T, Tadjkarimi S, Yacoub MH, Belvisi MG. Evidence for prejunctional muscarinic autoreceptors in human and guinea pig trachea. American journal of respiratory and critical care medicine 1995;152: 872-78.

16. Gallagher T, Maturo S, Fracchia S, Hartnick C. An analysis of children with tracheomalacia treated with ipratropium bromide (Atrovent). The Laryngoscope 2011; 121(S4S4):S211-S211.

\section{Hosted file}

Table.docx available at https://authorea.com/users/359754/articles/481572-a-retrospectiveanalysis-of-children-with-lower-airway-malacia-in-a-tertiary-paediatric-hospital

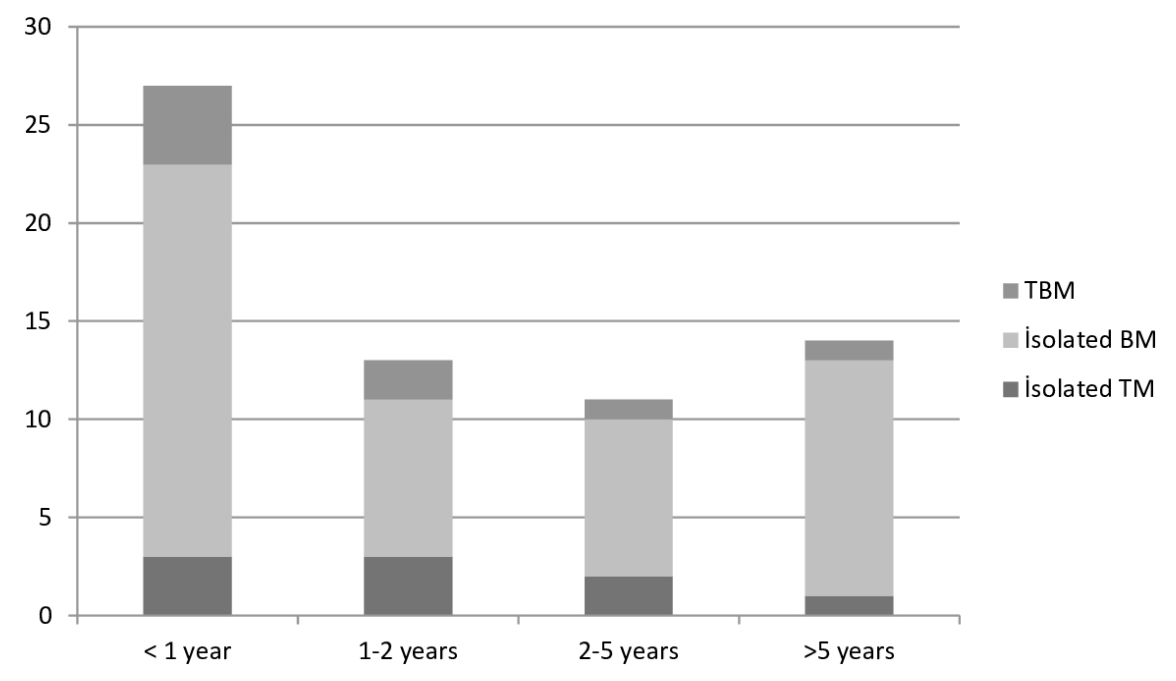

